$\Rightarrow$ FUNGAL GENETICS

\section{Fungal resistance needs a little extra}

Work in Judith Berman's laboratory at the University of Minnesota has identified common genome rearrangments associated with azole resistance in Candida albicans, according to a recent Science paper.

The C. albicans genome is known to tolerate aneuploidy - the gain or loss of chromosomes or chromosome fragments. Traditionally, changes in fungal karyotype are detected using CHEF (contour-clamped homogeneous electric field) whole-chromosome gels and quantitative Southern blots. However, the Berman laboratory recently adapted a technique, which was originally used to detect changes in the gene copy number at all loci in the Saccharomyces cerevisiae genome, to make it suitable for use in C. albicans. The technique involves comparative genome hybridization (CGH), in which digested genomic DNA from test and control isolates is differentially labelled with fluorescent dyes then hybridized to C. albicans whole-genome microarrays.

Anna Selmecki and Anja Forche from the Berman group used this CGH technique to take an in-depth look at the genome rearrangements present in clinical isolates of C. albicans resistant to the azole fluconazole. Aneuploidy was seven times more common in fluconazole-resistant isolates than in fluconazole-sensitive isolates, with chromosome 5 most frequently involved. Moreover, a specific segmental aneuploidy, an isochromosome comprising the two left arms of chromosome 5, was detected only in fluconazole-resistant isolates. The isochromosome, $\mathrm{i}(5 \mathrm{~L})$, was present both as an independent chromosome and fused to full-length chromosome 5 .

So, what genes could be responsible for the resistance? The left arm of chromosome 5 carries the ERG11 gene, which encodes the target of fluconazole, and TCA1, which encodes a transcription factor that upregulates the expression of an efflux pump on chromosome 3 , in addition to two ORFs encoding predicted efflux pumps. Analysis of the transcript profiles showed that the increased copy number associated with the aneuploidy correlates with increased gene expression.

Further analysis showed that strains carrying i(5L) had a growth advantage in the presence of fluconazole compared with strains lacking $\mathrm{i}(5 \mathrm{~L})$, and that the loss of $\mathrm{i}(5 \mathrm{~L})$ was accompanied by a concomitant loss in fluconazole resistance. The authors therefore suggest that agents that block or reduce isochromosome formation could be useful adjuncts to current azole therapeutic regimes.

Sheilagh Molloy, Senior Editor, Nature Reviews Microbiology

ORIGINAL RESEARCH PAPER Selmecki, A., Forche, A. \& Berman, J. Aneuploidy and isochromosome formation in drug-resistant Candida albicans. Science 313, 367-369 (2006)
Ethics watch

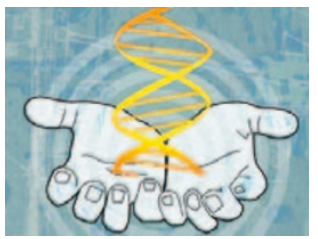

\section{PROMOTING EQUALITY AND DIVERSITY IN UK BIOMEDICAL AND CLINICAL RESEARCH}

There is growing evidence for population variation in susceptibility to disease and drug responses ${ }^{1,2}$. Therefore, widespread underrepresentation of ethnic minorities in UK biomedical and clinical research is surprising ${ }^{3,4}$. A recent Health Technology Assessment has found that flagship government and NHS (National Health Service) policy principles of equality and diversity are not formally promoted, despite concluding that estimates of efficacy and validity will be severely compromised if drugs are not tested on different socio-demographic groups ${ }^{3}$. With growing evidence of ethnic health inequalities ${ }^{5}$ it is imperative to understand and tackle the root causes. Apart from the ethical and social arguments, there are clear rational scientific, clinical, health and economic reasons to include different populations in research.

So why are minorities still underrepresented? Among the likely reasons is the lack of specific UK legislative or policy context. By contrast, the US Clinical Trials Fairness Act requires the inclusion of women and minorities in all state-funded research. The representation of both groups has increased since its introduction.

There are also the perceived problems of recruitment from minority ethnic communities who are generally considered 'hard-to-reach' and unwilling participants in clinical and biomedical research. However, recent evidence indicates that exclusion is the most probable cause of underrepresentation ${ }^{6}$. Additionally, critics argue that the associated costs of fairer inclusion make such research prohibitively expensive. Although these must be considered, costbenefit analyses should equally factor in the significant losses that are likely to be incurred through the development, delivery and uptake of ineffective, if not adverse, treatments and interventions. Minorities now constitute almost one-tenth of the UK population.

Widening minority ethnic group access to, and representation across, UK biomedical and clinical research will clearly, therefore, depend on a number of interdependent factors and processes. At the least it will require the development and delivery of a clear policy framework and its guidance, coupled with increased or better targeted resources. More accessible culturally and linguistically competent recruitment programmes are also needed. Establishing greater clarity about equality and diversity issues ${ }^{1}$ will be key to these developments. Among the most pressing issues is a need to: consistently and usefully define the UK's different populations and subpopulations; identify the contexts in which ethno- or population-specific data is actually important; and ascertain the relative sample sizes necessary to draw meaningful conclusions. The genetics community can play a significant part, and could therefore take a more active role in bringing about a change in policy and practice.

Increasing minority ethnic group representation is, however, only part of the battle. Such improvements will be of merit only if there is appropriate analysis and publication of the research findings. This is of more general concern as there is a wealth of unpublished information. But, if all requirements are met, the UK could be on course to fulfilling its commitment towards reducing health inequalities, and giving its disadvantaged populations a fairer deal.

Pritti Mehta, formerly of the Genetic Interest Group ( http://www.gig.org.uk) e-mail:pritti.mehta@homechoice.co.uk

ORIGINAL RESEARCH PAPERS ${ }^{1}$ Tishkoff, S. A. \& Kidd, K. K. Implications of biogeography of human populations for 'race' and medicine. Nature Genet. 36, S21-S27 (2004) | ${ }^{2}$ Tate, S. K. \& Goldstein, D.B. Will tomorrow's medicines work for everyone? Nature Genet. 36, S34-S42 (2004)|'Bartlett, C. et al. The causes and effects of socio-demographic exclusions from clinical trials. Health Technol. Assess. 9, 1-152 (2005)| ${ }^{4}$ Ranganthan, M., \& Bhopal, R. Exclusion and inclusion of nonwhite ethnic minority groups in 72 North American and European cardiovascular cohort studies. PLoS Med. 3, e44 (2006) | $\left.\right|^{5}$ Aspinall, P. \& Jacobson, B. Ethnic disparities in health and health care: a focused review of the evidence and selected examples of good practice. London Health Observatory [online], <http://www.lho.org.uk/Download/Public/8831/1/Ethnic Disparities_Report_4.pdf> (July 2004) $\left.\right|^{6}$ Wendler, D. et al. Are racial and ethnic minorities less willing to participate in health research? PLoS Med. 3, e19 (2006) 